\title{
Heavy flavour production measurements with ALICE at the LHC
}

\section{Francesco Bossù*, for the ALICE Collaboration}

iThemba Laboratory for Accelerator Based Sciences, Cape Town, South Africa

E-mail: bossultlabs.ac.za

Heavy quarks, i.e. charm and beauty, are produced in hard partonic scatterings, hence their measurement at the LHC has a twofold interest. On the one hand, heavy quarks are well suited to investigate the state of matter produced in heavy-ion collisions. On the other hand, the measurement of open heavy flavours in pp collisions is considered an optimal test-bench for perturbative QCD predictions. In the first two years of LHC operation, ALICE recorded data in pp collisions at $\sqrt{s}=2.76$ and $7 \mathrm{TeV}$, and in $\mathrm{Pb}-\mathrm{Pb}$ collisions at $\sqrt{s_{N N}}=2.76 \mathrm{TeV}$. Results of the data analysis both at mid-rapidity and at forward rapidity will be presented.

International Winter Meeting on Nuclear Physics,

21-25 January 2013

Bormio, Italy

${ }^{*}$ Speaker. 


\section{Introduction}

Quantum Chromodynamics (QCD) calculations on the lattice predict that, under extreme conditions of temperature and energy density, nuclear matter undergoes a phase transition to a state in which partons (quarks and gluons) are no longer confined in hadrons. Such a state is called Quark-Gluon Plasma (QGP). At the Large Hadron Collider (LHC), ultra-relativistic heavy-ions are made to collide in order to produce such a state of matter in the laboratory.

Heavy quarks (charm and beauty) are considered to be excellent probes of the medium formed in high-energy heavy-ion collisions. Indeed, due to their large masses, they are produced in the initial stage of the collision in hard partonic scattering processes. Before hadronizing into D and B mesons (containing charm and beauty quarks, respectively), charm and beauty quarks experience the full evolution of the produced medium. Furthermore, the measurement of the heavy flavour production cross section in proton-proton collisions is an important test-bench for perturbative QCD predictions.

If one considers nucleus-nucleus (A-A) collisions as an incoherent superposition of nucleon interactions, heavy flavour production is expected to scale with the number of nucleon-nucleon collisions. It is therefore possible to define an observable sensitive to any modifications of the expected yield. This observable is the nuclear modification factor and is defined as

$$
R_{A A}=\frac{1}{\left\langle N_{\text {coll }}\right\rangle} \frac{Y_{A A}}{Y_{p p}}
$$

where the yield $(Y)$ of heavy flavours in A-A collisions is compared to the one in proton-proton collisions multiplied by the mean number of binary nucleon-nucleon collisions $\left(N_{\text {coll }}\right)$ in A-A collisions. This last quantity can be estimated through the mean value of nuclear overlap function $\left(T_{A A}\right)$ in the Glauber model [1].

Since quarks and gluons carry colour charge, they are expected to lose energy via elastic collisions and via gluon radiation, while moving through a coloured medium such as the QGP. Due to their larger Casimir coupling, gluons should lose more energy with respect to quarks. In addition, the energy loss for quarks has a dependence on the mass through the so called "dead-cone" effect [2]: the heavier the quark is, the less energy it is expected to lose.

The parton energy loss modifies the transverse momentum $\left(p_{T}\right)$ spectra of the observed hadrons resulting in $R_{A A}<1$ in the intermediate and high $p_{\mathrm{T}}$ ranges. In addition, the colour charge and mass-dependence should give rise to the following $R_{A A}$ hierarchy: $R_{A A}^{\pi}<R_{A A}^{D}<R_{A A}^{B}$. Finally, one can check if the in-medium recombination is the predominant hadronization mechanism at low transverse momenta: in the D meson family, the relative abundance of strange over non-strange mesons is expected to be larger in A-A with respect to pp collisions [3].

Another important question related to heavy quarks concerns their participation in the collective motion of the medium and their thermalization in the medium. In non-central heavy-ion collisions, where an almond shaped fireball is formed, pressure gradients are larger in the plane defined by the beam direction and the impact parameter than in the direction perpendicular to that plane. One can therefore expect an anisotropy in the particle azimuthal distribution that can be decomposed in a Fourier series. The dominant anisotropic term in the Fourier decomposition is the second coefficient $v_{2}$, commonly called elliptic flow. If heavy quarks thermalise in the medium, a 
non-zero $v_{2}$ at low- $p_{T}$ is expected. At higher $p_{T}$, heavy quarks are not thermalised and are travelling for different path lengths in the medium, depending on the emission angle of the particle with respect to the reaction plane: the $v_{2}$ measurement at high- $p_{T}$ can give insights on the energy loss dependence on the in-medium path length.

\section{Heavy flavour measurements with ALICE}

The large heavy-flavour production cross section at LHC energies, which for charm (beauty) is about one (two) orders of magnitude larger than at RHIC, allows us to study the effects previously described with better precision and in a broader kinematical range. ALICE (A Large Ion Collider Experiment)[4] is the experiment at the LHC, which is designed to study heavy-ion collisions. It comprises a set of detectors covering the central rapidity region $(|\eta|<0.9)$ embedded in a solenoid magnet providing a $0.5 \mathrm{~T}$ field, a muon spectrometer at forward rapidity $(-4<\eta<-2.5)$ and a set of small acceptance detectors used for triggering and for the event characterization.

Heavy flavour measurements with ALICE can be carried out in different decay channels and kinematical regions. At mid-rapidity, heavy flavours can be measured by identifying the electrons from semi-leptonic decays as well as by reconstructing the full hadronic decays of charm mesons and baryons. At forward rapidity, the inclusive production of heavy flavours is measured in the semi-muonic decay channel.

\section{Heavy-flavour decay electrons}

Electron identification is performed at mid-rapidity $(|\eta|<0.6$ and $|\eta|<0.8$ depending on the detectors used in the analysis) combining the information of several Particle IDentification (PID) detectors. The Time Projection Chamber (TPC) is the main detector used both for the identification, via $\mathrm{d} E / \mathrm{d} x$ measurement, and for tracking. The information from the Time Of Flight (TOF) detector is combined with the TPC information for resolving PID ambiguities at low momenta, while at high momenta, the suppression of hadron contamination is performed adding the information of other detectors such as the Transition Radiation Detector (TRD) and the ElectroMagnetic Calorimeter (EMCal).

The most important background contributions to the inclusive single electron spectrum are: Dalitz decays of light neutral mesons, photon conversions in the material, di-electron decays of quarkonia and direct radiation. These background contributions were estimated either using a cocktail of the non-heavy-flavour electron sources, or by applying a selection on the invariant mass of $\mathrm{e}^{+} \mathrm{e}^{-}$pairs and they were statistically subtracted from the inclusive electron spectrum.

It is possible to separate the beauty contribution to the inclusive heavy flavour decay electron spectrum with two different techniques, one based on the angular correlation between electrons and hadrons and the second based on the larger decay length for B mesons compared to D mesons, which results in a larger separation of their decay electrons from the primary vertex compared to $\mathrm{D}$ mesons and other sources.

\section{D meson hadronic decays}

Thanks to the excellent tracking and vertexing capabilities of the TPC and the ITS (Inner Tracking System), at mid-rapidity charmed mesons can be reconstructed through their hadronic 
decay channels: $\mathrm{D}^{0} \rightarrow \mathrm{K}^{-} \pi^{+}, \mathrm{D}^{+} \rightarrow \mathrm{K}^{-} \pi^{+} \pi^{+}, \mathrm{D}^{*+} \rightarrow \mathrm{D}^{0} \pi^{+}, \mathrm{D}_{\mathrm{s}}^{+} \rightarrow \mathrm{K}^{+} \mathrm{K}^{-} \pi^{+}$and their charge conjugates. Thanks to the large decay length $(\approx 100-300 \mu \mathrm{m})$ of $\mathrm{D}$ mesons, charged charged tracks are selected which fulfill the decay topologies having the decay points displaced with respect to the interaction vertex. The $\mathrm{D}$ meson yield is then extracted from a fit to the invariant mass distribution of the selected candidates. The PID information from the TPC and the TOF helps in the reduction of the combinatorial background, which is particularly important in the high multiplicity environment of heavy-ion collisions.

\section{Heavy flavour decay muons}

At forward rapidity, the measurement of heavy flavours can be performed in the semi-leptonic decay channel by reconstructing single muon tracks in the muon spectrometer. It consists of a passive front absorber, five stations of tracking chambers equipped with a dipole magnet that provides $3 \mathrm{~T} \cdot \mathrm{m}$ of integrated B field and a trigger system placed after an iron filter. The muon identification is obtained by requiring the matching between the reconstructed track in the tracking system and the corresponding track segment in the trigger system. In order to remove fake tracks and tracks from beam-gas interactions, a cut on the transverse distance between the track and the interaction point is applied.

The main background contribution consists of in-flight muonic decays of pions and kaons. In pp collisions, this contribution is estimated with Monte Carlo simulations, while in $\mathrm{Pb}-\mathrm{Pb}$ events, where light hadron spectra can be modified by the medium formed in the collision, an extrapolation of $\pi$ and $\mathrm{K}$ yields measured at mid-rapidity is performed. Since the rapidity dependence of their yield in nuclear collisions is unknown, a $100 \%$ of systematic uncertainty is assigned to the extrapolated yields.

\section{Results}

The data samples used in the analyses presented in these proceedings have been recorded in 2010 and 2011 and they include pp collisions at $\sqrt{s}=2.76$ and $7 \mathrm{TeV}$ and $\mathrm{Pb}-\mathrm{Pb}$ collisions at $\sqrt{s_{N N}}=2.76 \mathrm{TeV}$. Similar trigger strategies have been used both for the pp and the $\mathrm{Pb}-\mathrm{Pb}$ cases: data have been collected with minimum bias triggers based on logical combinations of signals in VZERO detectors (two arrays of scintillators covering the pseudorapidity ranges $-3.7<\eta<-1.7$ and $2.8<\eta<5.1$ ) and the two innermost layers of the ITS. Additional specific trigger requirements have been set up for the electron and muon channels. The centrality of the collisions in $\mathrm{Pb}-\mathrm{Pb}$ events has been determined from the sum of the VZERO signal amplitudes.

\section{1 pp results}

Figure 1a shows the $p_{\mathrm{T}}$-differential cross section for inclusive heavy-flavour decay electrons in pp collisions at $\sqrt{s}=7 \mathrm{TeV}$ [5]. Minimum bias events, corresponding to an integrated luminosity of $2.6 \mathrm{nb}^{-1}$, were used. The ALICE measurement is complementary to that of the ATLAS collaboration [7], which covers a higher $p_{\mathrm{T}}$ range. FONLL perturbative QCD calculations describe well the data in all the measured $p_{\mathrm{T}}$ range [8]. A similar result has been obtained at forward rapidity in the semi-muonic decay channel [11] analyzing minimum bias events in coincidence with a single muon trigger signal using an integrated luminosity of $16.5 \mathrm{nb}^{-1}$. 


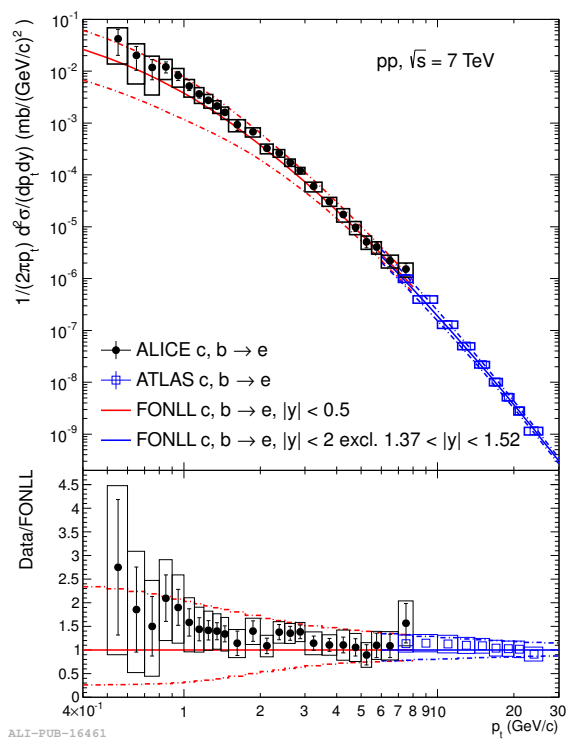

(a)

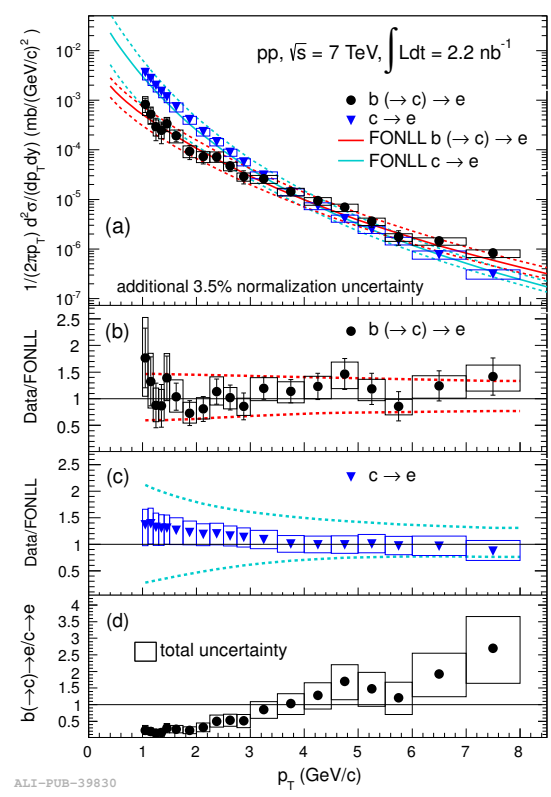

(b)

Figure 1: $p_{\mathrm{T}}$-differential cross section for heavy-flavour electron production at mid-rapidity for $\mathrm{pp}$ collisions at $\sqrt{s}=7 \mathrm{TeV}[5,6]$. The inclusive measurement (a) is shown together with the results from ATLAS. The contribution of electrons from B decays, shown together with the one from D decays, (b) is selected exploiting their separation from the primary vertex. The measurements are compared to FONLL pQCD calculations.

The separation of the beauty contribution to the single electron spectrum is shown in Figure $1 \mathrm{~b}$ based on an integrated luminosity of $2.2 \mathrm{nb}^{-1}$ [6]. The transverse momentum spectra of the beauty and the charm components are well reproduced by pQCD calculations and their ratio shows that the beauty decay contribution to the inclusive electron yield takes over the charm one for $p_{\mathrm{T}}>4$ $\mathrm{GeV} / c$.

Perturbative QCD calculations also describe within uncertainties the measurements of the inclusive production cross sections for prompt $\mathrm{D}$ mesons in pp collisions at $\sqrt{s}=7 \mathrm{TeV}[12,13]$. Figure 2 shows the $p_{\mathrm{T}}$-differential cross section for $\mathrm{D}^{+}$and $\mathrm{D}_{\mathrm{s}}^{+}$mesons and similar results have been obtained for the $\mathrm{D}^{0}$ and the $\mathrm{D}^{*+}$ mesons. The determination of the total cē production cross section has been performed by extrapolating the measured $p_{\mathrm{T}}$-differential cross sections to the full phase space [14].

The same analysis has been performed on the data collected in pp collision at $\sqrt{s}=2.76 \mathrm{TeV}$. Results for muons and prompt D mesons $[15,14]$ and preliminary results for heavy-flavour decay electrons are reproduced by pQCD calculations within uncertainties.

\section{2 $\mathrm{Pb}-\mathrm{Pb}$ results}

The heavy flavour nuclear modification factor has been measured in different centrality classes in all the hadronic and semi-leptonic channels previously described [15, 16, 18]. 


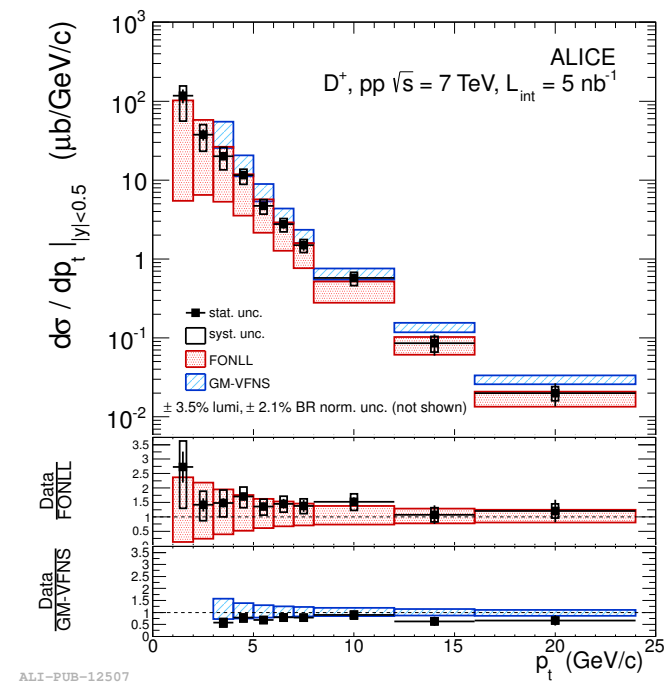

(a)

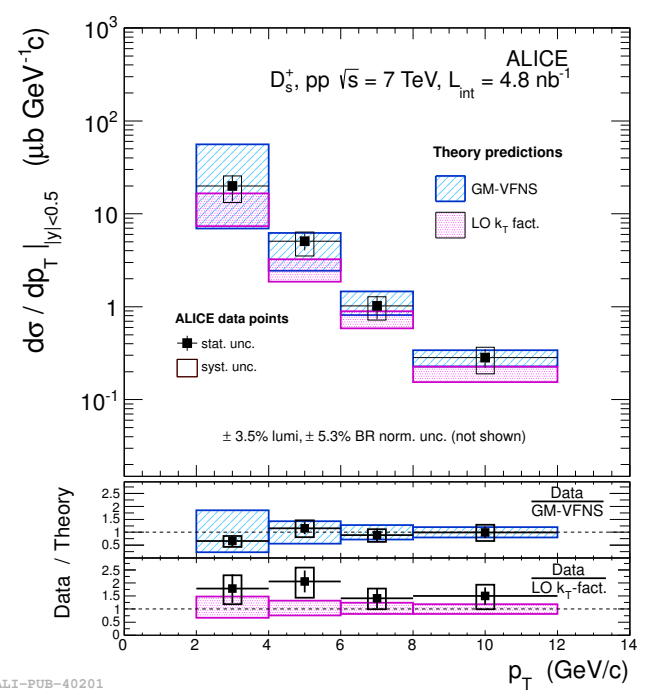

(b)

Figure 2: $\quad p_{\mathrm{T}}$-differential cross sections of $\mathrm{D}^{+}$and $\mathrm{D}_{\mathrm{s}}^{+}$at mid-rapidity in pp collisions at $\sqrt{s}=$ $7 \mathrm{TeV}[12,13]$ compared to FONLL[8], GM-VFNS[10] and LO- $k_{t}$-factorization[9] theoretical predictions.

In Figure 3a, $R_{A A}$ 's for electrons and muons from semi-leptonic decay of heavy flavours in the most central collisions (0-10\%) are shown as a function of the transverse momentum. The protonproton reference used for the muon $R_{A A}$ is the cross section measured in pp collisions at $\sqrt{s}=2.76$ $\mathrm{TeV}$, while for the electron channel the reference has been obtained, for $p_{\mathrm{T}}<8 \mathrm{GeV} / c$, by rescaling in energy the cross section measured at $\sqrt{s}=7 \mathrm{TeV}$. The scaling factor was defined as the ratio of the FONLL predictions at the two energies [17]. For $p_{\mathrm{T}}>8 \mathrm{GeV} / c$ FONLL predictions were used as pp reference in the $R_{A A}$. Despite the different rapidity intervals, muons and electrons show similar suppression and $p_{\mathrm{T}}$ dependence.

The nuclear modification factor for prompt $\mathrm{D}$ mesons in the most central collisions (0-7.5\%) is shown in Figure 3b. This includes the first measurement of $\mathrm{D}_{\mathrm{s}}^{+}$in heavy ion collisions. As for the heavy flavour decay electrons, the pp reference has been extrapolated from the measurement at $\sqrt{s}=7 \mathrm{TeV}$ through pQCD FONLL calculations. The results for $\mathrm{D}^{+}, \mathrm{D}^{0}, \mathrm{D}^{*+}$ are compatible and show a suppression by a factor 3-5 for $p_{\mathrm{T}}>5 \mathrm{GeV} / c$. The measurement for $\mathrm{D}_{\mathrm{s}}^{+}$seems to indicate a higher $R_{A A}$ value with respect to non-strange $\mathrm{D}$-meson species at low $p_{\mathrm{T}}$, but the large uncertainties do not allow a firm conclusions on this issue yet.

In order to study the expected hierarchy in the energy loss of light flavour, charm and beauty hadrons, Figure 4 a shows the average $R_{A A}$ of $\mathrm{D}$ mesons $\left(\mathrm{D}^{+}, \mathrm{D}^{0}\right.$ and $\left.\mathrm{D}^{*+}\right)$ as a function of the transverse momentum together with $R_{A A}$ of charged particles and pions in the most central collisions. The two nuclear modification factors have a similar $p_{\mathrm{T}}$ dependence. A hint of a larger magnitude for $R_{A A}$ od $\mathrm{D}$ mesons is observed at low $p_{\mathrm{T}}$, but more accurate studies with larger statistics are needed to draw firm conclusions.

In Figure $4 \mathrm{~b}, R_{A A}$ of D mesons in $6<p_{\mathrm{T}}<12 \mathrm{GeV} / \mathrm{c}$ is shown as a function of the centrality. The result of $\mathrm{J} / \psi$ from B decay (non-prompt $\mathrm{J} / \psi$ ) measured by the CMS collaboration at mid- 


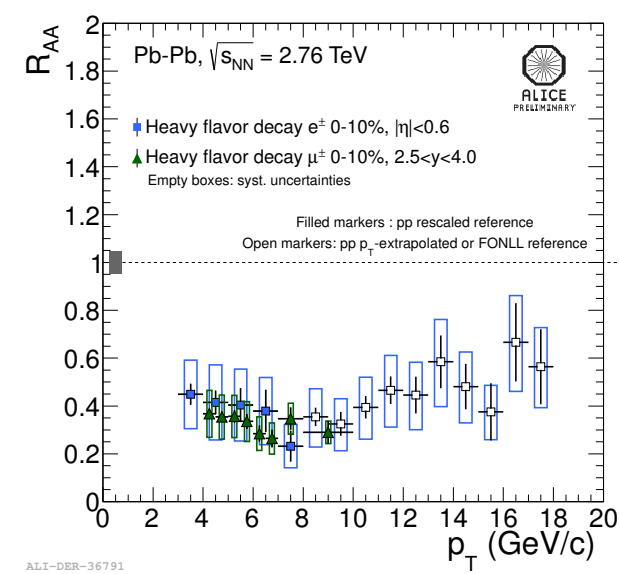

(a)

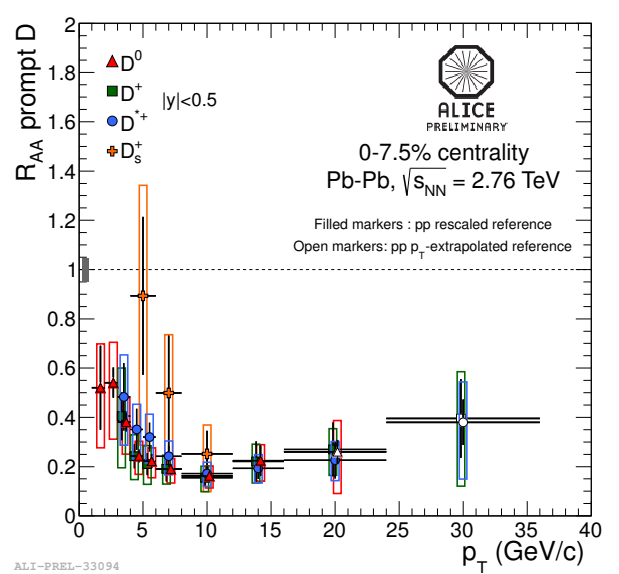

(b)

Figure 3: $\quad R_{A A}$ as a function of $p_{\mathrm{T}}$ for central $\mathrm{Pb}-\mathrm{Pb}$ collisions at $\sqrt{s_{N N}}=2.76 \mathrm{TeV}$. (a): the measurement in the electron channel at mid-rapidity is shown together with the one in the muon decay channel at forward rapidity. (b): prompt $\mathrm{D}^{+}, \mathrm{D}^{0}, \mathrm{D}^{*+}$ and $\mathrm{D}_{\mathrm{S}}^{+} R_{A A}$ at mid-rapidity. The global systematic uncertainties are shown as filled boxes at $R_{A A}=1$.

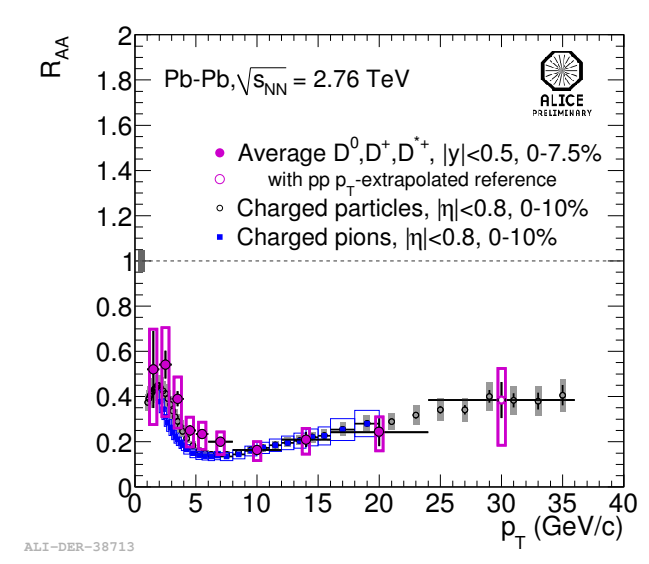

(a)

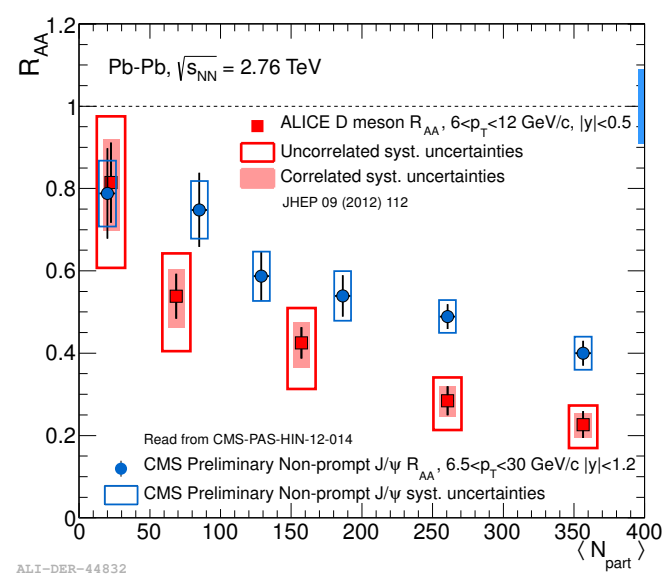

(b)

Figure 4: (a): average $\mathrm{D}^{+}, \mathrm{D}^{0}$ and $\mathrm{D}^{*+}$ mesons in the $0-7.5 \%$ centrality class and charged hadron and pion $R_{A A}$ in the $0-10 \%$ centrality class. Error bars indicate the statistical uncertainties, empty boxes the systematic uncertainties. (b): average D meson $R_{A A}\left(6<p_{\mathrm{T}}<12 \mathrm{GeV} / c\right)$ as a function of the number of participant nucleons, $\mathrm{N}_{\text {part }}$ compared with the CMS measurement of non-prompt $\mathrm{J} / \psi$ measured in $|y|<1.2\left(6.5<p_{\mathrm{T}}<30 \mathrm{GeV} / c\right)$.

rapidity $(|y|<1.2)$ and $p_{\mathrm{T}}>6.5 \mathrm{GeV} / c$ is also plotted in the figure for comparison. The $R_{A A}$ of non-prompt $\mathrm{J} / \psi$ is larger than the one of $\mathrm{D}$ mesons, which supports the expected hierarchy in the energy loss of $b$ and $c$ quarks.

The second term $v_{2}$ of the Fourier decomposition of the azimuthal distribution of heavy flavours, the so-called elliptic flow, has been measured at mid-rapidity in the electron decay channel (Fig- 


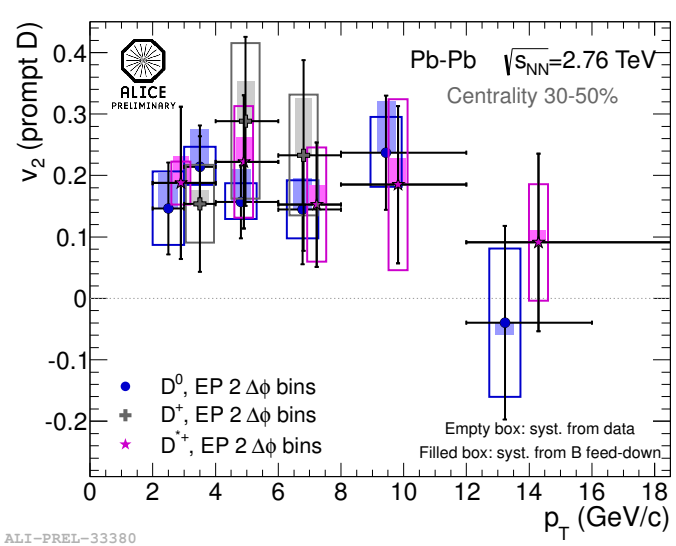

(a)

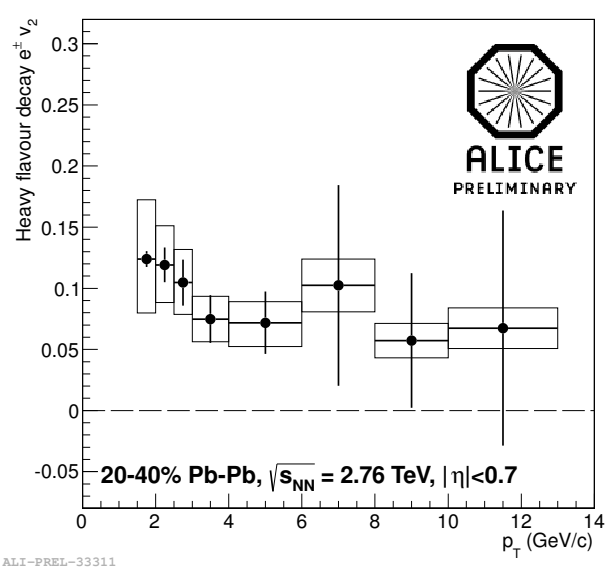

(b)

Figure 5: Heavy flavour elliptic flow in semi-central $\mathrm{Pb}-\mathrm{Pb}$ collisions at $\sqrt{s_{N N}}=2.76 \mathrm{TeV}$ as function of the transverse momentum (a) for prompt D mesons and (b) heavy flavour decay electrons. Error bars represent statistical uncertainties and boxes indicate systematic errors.

ure 5b) and for prompt $\mathrm{D}^{+}, \mathrm{D}^{0}$ and $\mathrm{D}^{*+}$ mesons (Figure 5a) [18]. The measurements have been performed for semi-central $\mathrm{Pb}-\mathrm{Pb}$ collisions, namely in the $20-40 \%$ centrality range for electrons and $30-50 \%$ for $\mathrm{D}$ mesons. The elliptic flow has been measured as a function of the transverse momentum in the range $1.5<p_{\mathrm{T}}<13 \mathrm{GeV} / c$ for the electrons and $2<p_{\mathrm{T}}<18 \mathrm{GeV} / c$ for the charmed mesons. The $v_{2}\left(p_{\mathrm{T}}\right)$ of the three $\mathrm{D}$ meson species agrees within uncertainties. All measurements show a non-zero elliptic flow at low $p_{\mathrm{T}}$ which suggests that heavy quarks participate in the collective expansion of the produced medium.

\section{Conclusions}

With the analysis of the data collected both in $\mathrm{pp}$ and in $\mathrm{Pb}-\mathrm{Pb}$ collisions in the first years of LHC operation, the ALICE collaboration has shown that heavy flavour hadrons and leptons from their decay provide valuable information to understand heavy quark production in hadronic collisions and their interaction with the quark-gluon plasma.

The cross sections of charmed hadrons and heavy flavour (charm and beauty) decay leptons have been measured in pp collisions at $\sqrt{s}=2.76 \mathrm{TeV}$ and $\sqrt{s}=7 \mathrm{TeV}$. Perturbative QCD calculations describe the measurements within uncertainties. Proton-proton results provide the reference for the $\mathrm{Pb}-\mathrm{Pb}$ results. In the most central collisions, the heavy flavour measurements, at midrapidity in the electron decay channel and for prompt $\mathrm{D}$ mesons, and at forward rapidity in the muon decay channel, show a suppression of the yield by a factor 3-4 in the intermediate $p_{\mathrm{T}}$ region. For a better understanding of the hot medium effects, analyses of proton-nucleus collisions, recorded early in 2013, are ongoing with the aim of measuring the effect of the cold nuclear matter on heavy-flavour production.

Charmed mesons and heavy-flavour electrons exhibit a positive elliptic flow in semi-central collisions possibly indicating that heavy quarks participate in the collective motion of the system. 
These results underline the importance of high precision heavy flavour studies in the next LHC runs.

\section{References}

[1] M. L. Miller, K. Reygers, S. J. Sanders and P. Steinberg, Glauber modeling in high energy nuclear collisions, Ann. Rev. Nucl. Part. Sci. 57 (2007) 205 [nucl-ex/0701025].

[2] Y. L. Dokshitzer and D. E. Kharzeev, Heavy quark colorimetry of QCD matter, Phys. Lett. B 519 (2001) 199 [hep-ph/0106202]

[3] Y. L. Dokshitzer and D. E. Kharzeev, Heavy quark colorimetry of QCD matter, Phys. Lett. B 519, 199 (2001) [hep-ph/0106202].

[4] K. Aamodt et al. [ALICE Collaboration], The ALICE experiment at the CERN LHC, JINST 3 (2008) S08002

[5] B. Abelev et al. [ALICE Collaboration], Measurement of electrons from semileptonic heavy-flavour hadron decays in pp collisions at $\sqrt{s}=7 \mathrm{TeV}$, Phys. Rev. D 86 (2012) 112007 [arXiv:1205.5423 [hep-ex]].

[6] B. Abelev et al. [ALICE Collaboration], Measurement of electrons from beauty hadron decays in $p p$ collisions at $\sqrt{s}=7 \mathrm{TeV}$, Phys. Lett. B 721 (2013) 13 [arXiv:1208.1902 [hep-ex]].

[7] G. Aad et al. [ATLAS Collaboration], Measurements of the electron and muon inclusive cross-sections in proton-proton collisions at $\sqrt{s}=7 \mathrm{TeV}$ with the ATLAS detector, Phys. Lett. B 707 (2012) 438 [arXiv:1109.0525 [hep-ex]].

[8] M. L. Mangano, P. Nason and G. Ridolfi, Heavy quark correlations in hadron collisions at next-to-leading order, Nucl. Phys. B 373 (1992) 295.

[9] R. Maciula and A. Szczurek, Open charm production at the LHC - $k_{t}$-factorization approach, arXiv:1301.3033 [hep-ph].

[10] B. A. Kniehl, G. Kramer, I. Schienbein and H. Spiesberger, Inclusive Charmed-Meson Production at the CERN LHC, Eur. Phys. J. C 72 (2012) 2082 [arXiv:1202.0439 [hep-ph]].

[11] B. Abelev et al. [ALICE Collaboration], Heavy flavour decay muon production at forward rapidity in proton-proton collision at $\sqrt{s}=7 \mathrm{TeV}$, Phys. Lett. B 708 (2012) 265 [arXiv:1201.3791 [hep-ex]].

[12] B. Abelev et al. [ALICE Collaboration], Measurement of charm production at central rapidity in proton-proton collisions at $\sqrt{s}=7 \mathrm{TeV}$, JHEP 1201 (2012) 128 [arXiv:1111.1553 [hep-ex]].

[13] B. Abelev et al. [ALICE Collaboration], $D_{s}^{+}$meson production at central rapidity in proton-proton collisions at $\sqrt{s}=7 \mathrm{TeV}$, Phys. Lett. B 718 (2012) 279 [arXiv:1208.1948 [hep-ex]].

[14] B. Abelev et al. [ALICE Collaboration], Measurement of charm production at central rapidity in proton-proton collisions at $\sqrt{s}=2.76 \mathrm{TeV}$, JHEP 1207 (2012) 191 [arXiv:1205.4007 [hep-ex]].

[15] B. Abelev et al. [ALICE Collaboration], Production of muons from heavy flavour decays at forward rapidity in pp and Pb-Pb collisions at $\sqrt{s_{N N}}=2.76 \mathrm{TeV}$, Phys. Rev. Lett. 109, 112301 (2012) [arXiv:1205.6443 [hep-ex]].

[16] B. Abelev et al. [ALICE Collaboration], Suppression of high transverse momentum D mesons in central Pb-Pb collisions at $\sqrt{s_{N N}}=2.76 \mathrm{TeV}$, JHEP 1209 (2012) 112 [arXiv:1203.2160 [nucl-ex]]. 
[17] R. Averbeck, N. Bastid, Z. C. del Valle, P. Crochet, A. Dainese and X. Zhang, Reference Heavy Flavour Cross Sections in pp Collisions at $\sqrt{ } s=2.76 \mathrm{TeV}$, using a $p Q C D$-Driven $\sqrt{ } s$-Scaling of ALICE Measurements at $\sqrt{ } s=7 \mathrm{TeV}$, arXiv:1107.3243 [hep-ph].

[18] Z. C. del Valle [ALICE Collaboration], Heavy-flavor suppression and azimuthal anisotropy in $\mathrm{Pb}-\mathrm{Pb}$ collisions at $\sqrt{\left(s_{N N}\right)}=2.76 \mathrm{TeV}$ with the ALICE detector, arXiv:1212.0385 [nucl-ex]. 